\title{
Long-term Outcomes of Catheter Ablation for Ventricular Arrhythmias in Post-Myocarditis Patients: Insights from a Meta-Analysis of Current Data
}

\author{
Emmanuel Androulakis ${ }^{1}$. Debbie Falconer ${ }^{2} \cdot$ Alexandros Briasoulis $^{3}$. Catrin Sohrabi ${ }^{4} \oplus$ - Wei-Yao Lim $^{4}$. \\ Gerasimos Siasos $^{5} \cdot$ Nikhil Ahluwalia $^{4} \cdot$ Adam Graham $^{4} \cdot$ Nikolaos Papageorgiou $^{4,6}$
}

Accepted: 7 February 2022 / Published online: 19 February 2022

(c) The Author(s), under exclusive licence to Springer Nature Switzerland AG 2022

\begin{abstract}
Background In the past decade, catheter ablation (CA) has become a rapidly expanding treatment option for ventricular tachycardia (VT); however it is not commonly utilised for patients with post-myocarditis VT. We aimed to systematically review up-to-date evidence regarding feasibility, effectiveness, and safety of CA, with a specific focus on long-term relapse rate and procedural complications.

Methods A structured electronic database search (PubMed, Embase, Cochrane) of the scientific literature was performed according to PRISMA guidelines for studies describing outcomes at up to 7.3 years after CA. The primary outcome measured was VT recurrence post-ablation. Procedural success was defined as freedom of ventricular arrhythmias (at the end of follow-up after an ablation procedure). The secondary outcome was significant procedural complications which included procedural death, stroke, cardiac tamponade, acute myocardial infarction, major vascular complications, and major bleeding, assessed on a study-by-study basis.

Results A total of 186 patients were included in analysis with most patients (88\%) being male. Over the follow-up period, there was a $18 \%$ relapse rate $(n=34)$ (confidence interval $(\mathrm{CI}) ; 0.12-0.24, I^{2} \approx 0, p=0.77$ ) with the majority of patients remaining VT free for the duration of follow-up. The overall procedural complication rate was $3.0 \%(n=7)(\mathrm{CI} ; 0.01-0.07$, $I^{2} \approx 0, p=0.44$ ), and of note, there were no peri-procedural deaths or heart transplant surgeries reported. However, a single study reported a mortality of $10 \%(n=2)$ during the follow-up period.

Conclusions CA is an effective and durable long-term therapeutic strategy for post-myocarditis VT patients with limited relapse rate and very low complication rates based on these non-randomised data. Larger randomised-controlled trials with standardised treatment and long follow-up are required to compare CA versus conventional treatment in the post-acute myocardial phase.
\end{abstract}

Keywords Catheter ablation $\cdot$ Ventricular tachycardia $\cdot$ Myocarditis $\cdot$ Outcomes

Emmanuel Androulakis and Debbie Falconer contributed equally.

Nikolaos Papageorgiou

drnpapageorgiou@yahoo.com

1 Royal Brompton \& Harefield NHS, Heart Imaging Centre, London, UK

2 Cardiology department, Queens Hospital, Romford, UK

3 Section of Heart Failure and Transplant Division of Cardiovascular Diseases, University of Iowa Hospitals and Clinics, Iowa City, IA, USA
4 Electrophysiology Department, Barts Heart Centre, St. Bartholomew's Hospital, West Smithfield, London EC1A 7BE, UK

5 3Rd Department of Cardiology, Sotiria Hospital University of Athens Medical School Athens, Athens, Greece

6 Institute of Cardiovascular Science, University College London, London, UK 


\section{Introduction}

Myocarditis, defined as inflammation of the heart muscle, can arise from a variety of infectious agents, autoimmune processes, or drugs [1]. More recently, severe acute respiratory coronavirus 2 (SARS-CoV2) has been associated with myocarditis, though the exact mechanism behind this is not fully understood [2].

Arrhythmias such as ventricular tachycardia (VT) can occur during acute myocarditis (AM), or persist chronically after the acute episode 'post-myocarditis' (PM) even in the presence of a normal left ventricular ejection fraction (LVEF). Arrhythmias in later stage myocarditis may result from chronic inflammation, where there is no recovery from the initial inflammatory phase, or secondary to post-inflammatory myocardial scar. In the post-inflammatory phase, fibrosis results from healing of inflamed myocardial tissue, which can lead to scar-related arrhythmogenesis [3]. Current guidelines recommend antiarrhythmic therapy (generally amiodarone) first line to suppress these arrhythmias. Implantable cardiac defibrillator (ICD) insertion is advised where pharmacotherapy is unsuccessful at controlling arrhythmias [4].

Catheter ablation is not currently recommended for patients with PM ventricular arrhythmias. However, it has already demonstrated promise in patients with Chagasic cardiomyopathy, a major cause of non-ischaemic cardiomyopathy in south America, in which the incidence of VT and sudden cardiac death is high despite treatment with amiodarone [5].

There is growing interest in the role of ablation to treat VT in the PM phase, as an adjunct or alternative to current treatment strategies. This report analyses the most up-to-date evidence regarding feasibility, effectiveness, and safety of catheter ablation, with a specific focus on long-term relapse rate and procedural complications.

\section{Methods}

We systematically searched the electronic databases MEDLINE, PUBMED, EMBASE, and the Cochrane Library for Central Register of Clinical Trials, using the MESH terms 'post-myocarditis' OR 'after myocarditis' AND 'catheter ablation' OR 'radiofrequency ablation' AND 'ventricular tachycardia' OR 'ventricular arrhythmia'. We limited our search to studies on adult human subjects and published in English language peer-reviewed journals until March 2021. The population of interest included patients with medication-refractory ventricular tachycardia which persisted or post-myocarditis. Studies were selected according to PRISMA guidelines. The literature search and study selection took place from September 2020 to March 2021. Analyses were performed using STATA ${ }^{\circledR}$ (version IC 16.1, StataCorp LLC). Continuous variables are presented as mean $\pm \mathrm{SD}$ or median and interquartile ranges (IQRs).

The primary outcome measured was VT recurrence post-ablation. Procedural success was defined as freedom of ventricular arrhythmias (at the end of follow-up after an ablation procedure). The secondary outcome was significant procedural complications which included procedural death, stroke, cardiac tamponade, acute myocardial infarction, major vascular complications, and major bleeding, assessed on a study-by-study basis. Bias assessment was performed with the quality assessment scale for case series studies (Suppl. Table).

\section{Study Selection}

We included prospective studies and retrospective cohorts published as original articles in peer-reviewed scientific journals in English. We excluded those trials that did not report any of the following variables or outcomes: number of adverse events, length of study, or description of the main relevant features of the study population including gender, age, description of the procedure, and concomitant therapy. We did not restrict eligibility according to renal function.

The data was independently extracted by two authors using standardised protocol and reporting form. Two independent reviewers (E.A. and D.F.) screened all abstracts and titles to identify potentially eligible studies. The full text of these potentially eligible studies was then evaluated. Agreement of at least two reviewers was required for decisions regarding inclusion or exclusion of studies. Studies that full text was not available were excluded from the analysis.

\section{Results}

\section{Study Selection and Patient Characteristics}

A total of five studies met our eligibility criteria and were included. The selection process is illustrated in Fig. 1 (PRISMA). A total of 186 patients were included in analysis with most patients (88\%) being male. From the four studies who reports this information, $50 \%$ of patients had an ICD in place and $70.4 \%$ of the patients were on anti-arrhythmic drugs. Baseline data and the design of selected studies are summarised in Table 1. Out of the five studies in the analysis, four were retrospective case series with one prospective case series. All studies except one were single-centre observational studies. 
Fig. 1 PRISMA flow-diagram

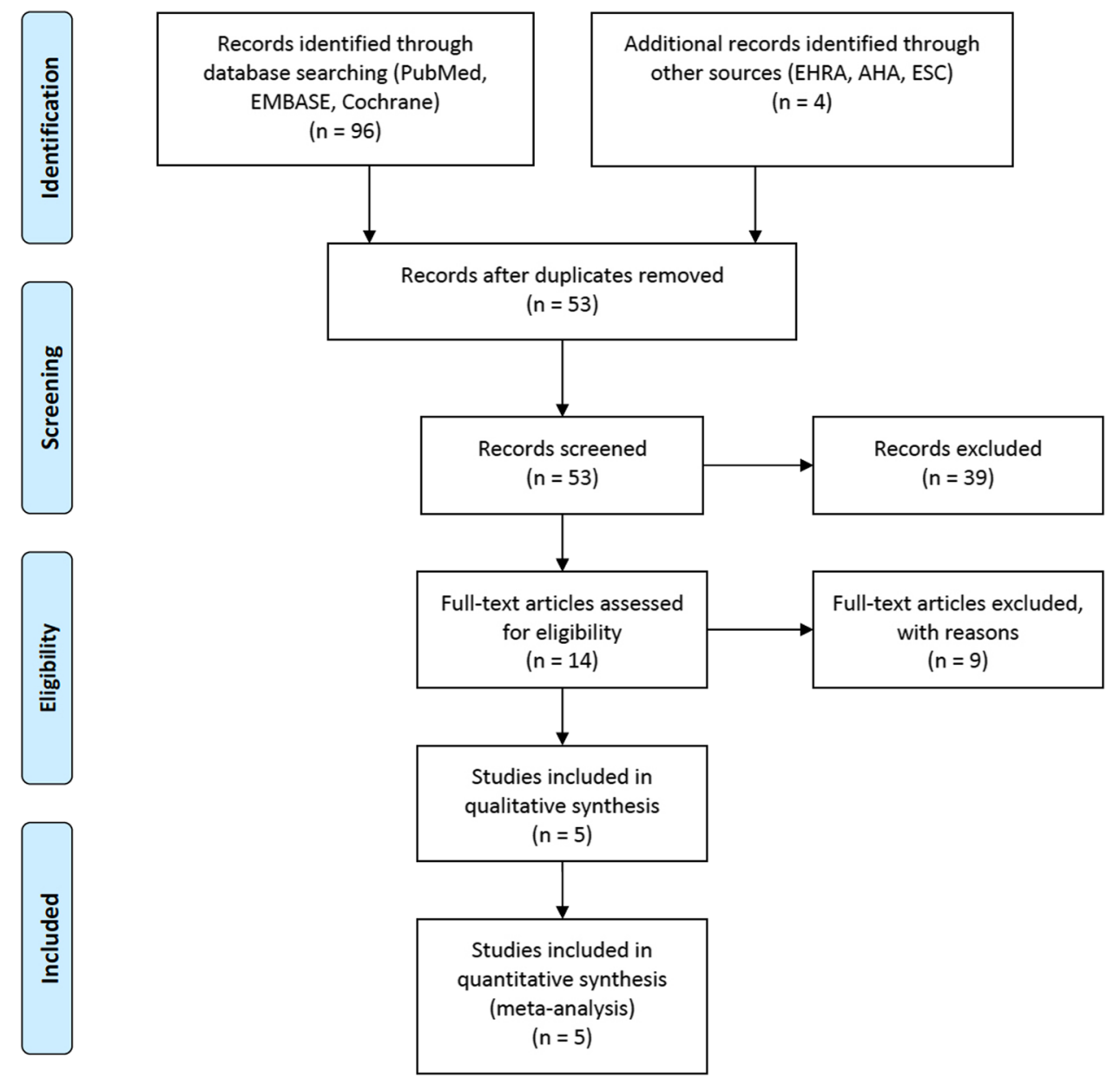

\section{Procedural Data}

Of the five studies, four reported the mean procedure time whilst one study only reported radiofrequency time and screening times. The mean procedural time was $272 \mathrm{~min}$. All studies used Biosense Websters's CARTO mapping system, and four out of the five studies also used Abbot's Ensite/NavX systems. Seventy-three per cent of patients had a combined endo-epicardial mapped performed during ablation. All studies performed substrate modification, and mapping in VT was only performed if hemodynamically tolerated. Table 2 prov

ides a summary of procedural data obtained from all five studies.

\section{Efficacy and Complications}

The median follow-up period ranged from 23 to 63 months. Over the follow-up period, there was an $18 \%$ relapse rate ( $n=34$ ) (confidence interval (CI); 0.12-0.24, $I^{2} \approx 0, p=0.77$ ) with the majority of patients remaining VT free for the duration of follow-up (Fig. 2). Only one study recorded the percentage of re-do procedures. The overall procedural complication rate was $3.0 \%\left(n=7, \mathrm{CI} ; 0.01-0.07, I^{2} \approx 0\right.$, $p=0.44$ ), and of note, there were no peri-procedural deaths or heart transplant surgeries reported. However, a single study reported a mortality of $10 \%(n=2)$ during the followup period (Fig. 3). Table 2 provides a summary of the efficacy and complications recorded.

\section{Discussion}

This important set of data, although not large in numbers given current limited literature, suggests catheter ablation may be an effective treatment for VT in the post-myocarditis phase for patients with preserved LVEF, with low relapse rates post-procedure. Importantly, when carried out in specialist centres, it is a safe procedure with low rates of procedural complications. More specifically, Vaseghi et al. showed that catheter ablation in patients post-myocarditis had lower rates of VT recurrence when compared with patients with hypertrophic cardiomyopathy, valvular cardiomyopathy, or sarcoidosis [6].

In PM patients, there is a high incidence of intramural and subepicardial disease, and an epicardial approach would be 


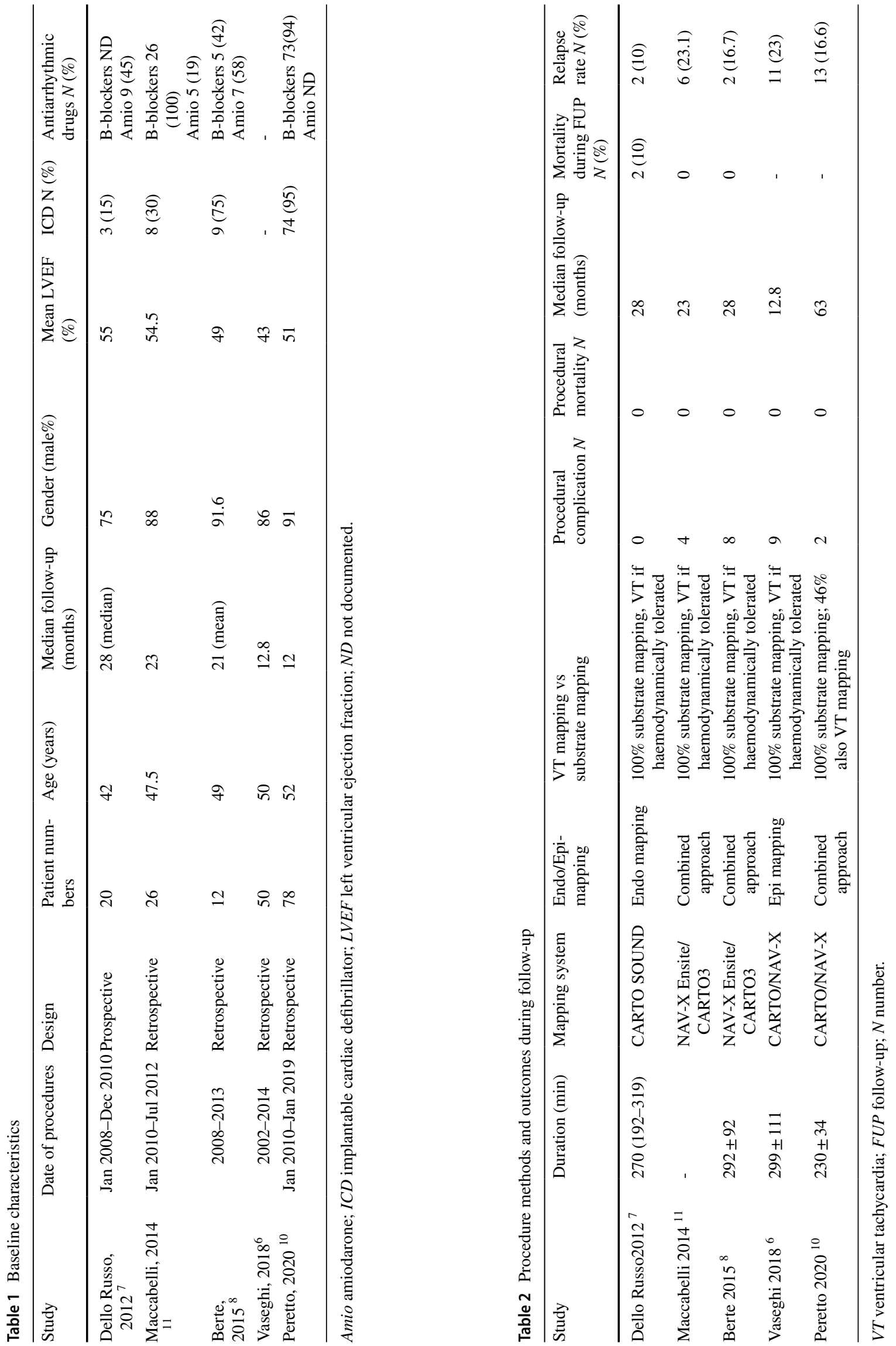




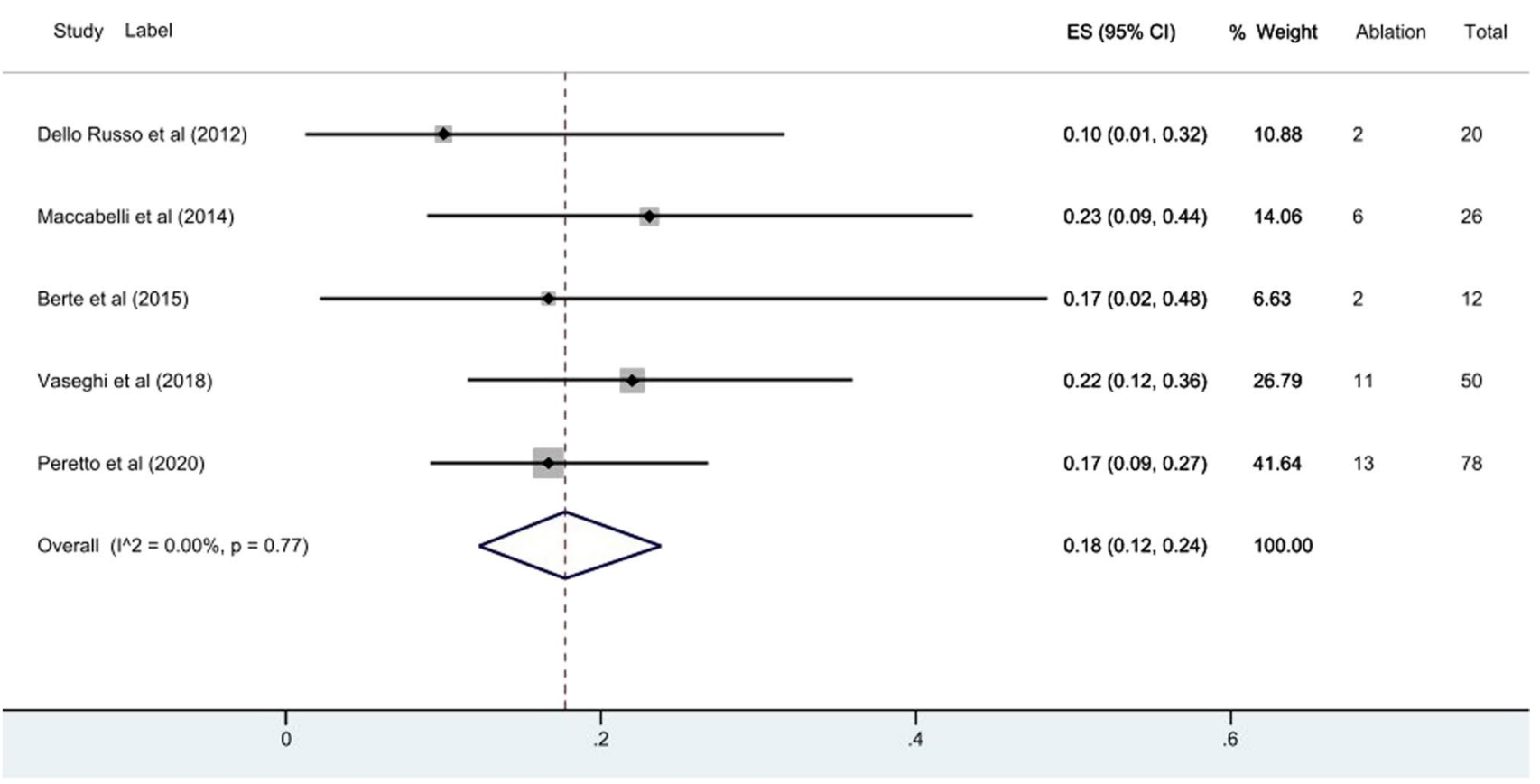

Fig. 2 Overall relapse rates during follow-up

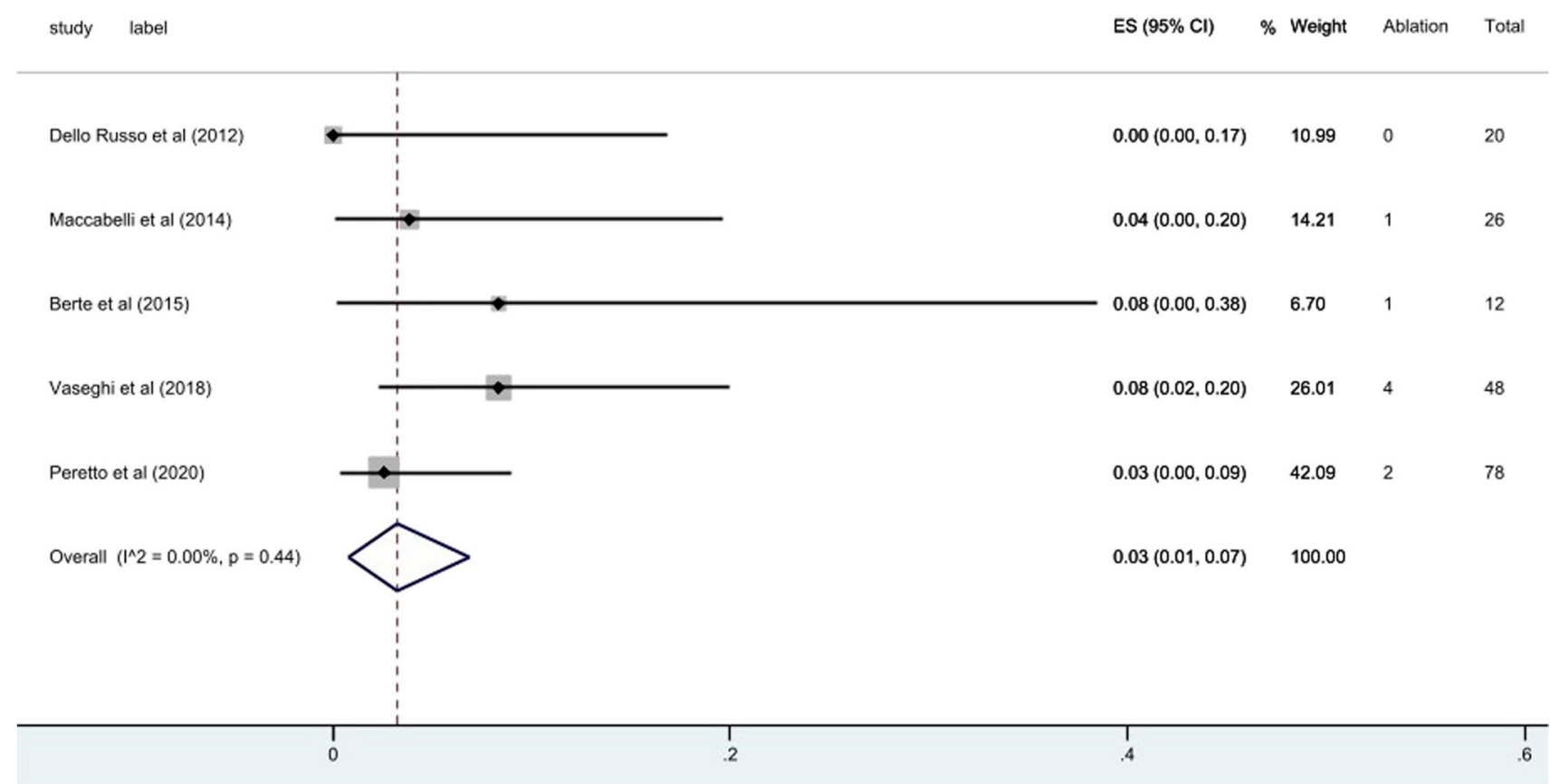

Fig. 3 Overall procedural complication rates

more suitable for some patients first line [7, 8]. Dello Russo et al. offered endocardial approaches to all patients in the first instance, followed by an epicardial approach if unsuccessful, exposing the patient to procedural risks twice [7], whilst over $90 \%$ of patients reported in Berte et al. were offered a combined approach in the first instance [8]. It should be noted that
Dello Russo performed the earliest study in 2012. All studies performed later offered a combined or epicardial approach. However, the need for an epicardial approach means the procedure must be carried out in specialist centres.

Patient selection for which approach is more suitable can be guided by CMR, for pre-procedural scar location, 
further improving procedural success [9]. Should this be performed as standard, procedural success and relapse rate may be improved further. A barrier to performing larger trials is that myocarditis can go undiagnosed in the acute setting due to its variety of presentations. Patients can present later with arrhythmias in the PM phase, but without a biopsy or imaging-guided diagnosis in the first instance, they may not be included in the post-myocarditis cohort for trials. Interestingly, Perreto compared the effectiveness of catheter ablation in the acute and PM phases and found that ablation was a much more effective treatment in the PM phase. Actually, catheter ablation in the acute myocarditis phase was the only predictor of VT recurrences at 12 months (hazard ratio: 9.5) [10].

Most patients included were on anti-arrhythmic medication at the time of ablation. To our knowledge, there is no data directly comparing anti-arrhythmic medication and ablation as treatment for VT in this setting. This data suggests ablation may be effective in preventing VT recurrence as an adjunct to anti-arrhythmic medication but are unlikely to replace pharmacotherapy as a first-line treatment. However, following ablation patients may be able to reduce the dose of antiarrhythmic medications, particularly pertinent when using amiodarone which long term can cause hepatotoxicity and thyroid dysfunction and is contraindicated in pregnancy.

Around $50 \%$ of patients already had an ICD in situ. Maccabelli et al. noted that 8 of 26 patients had no ICD inserted at the end of their follow up period with no recurrence of arrhythmia [11]. The negative impact of recurrent ICD shocks on a patient's morbidity and mortality is widely acknowledged. Ablation could be used to reduce the burden of ICD therapies, or even negate the need to implant a device in selected patients, thus significantly improving quality of life. Given the safety of the procedure and low complication rates, catheter ablation could be used early in the course of the disease, rather than delaying the procedure until the patient is on maximal medical therapy with an ICD in situ.

Larger, randomised-controlled trials with standardised treatment and follow-up regimens are required to compare VT relapse rates in those who undergo ablation and patients who are managed with conventional treatment alone before any changes are made to guidelines. However, this early data suggest catheter ablation is a viable option for patients with PM ventricular arrhythmias and could afford patients more choice of potential treatments.

\section{Limitations}

All studies are observational, small (with $<100$ patients per cohort) and mostly single centre. The studies lack control groups to allow for comparison of relapse rates between those who undergo ablation, and those who do not. The baseline characteristics reported in the studies focus on cardiac parameters such as ejection fraction and scar burden. There is little detail on other past medical history, and it is likely patients with good functional baselines and few comorbidities were selected, and thus these early results will not be generalisable to a significant portion of the population. There is also heterogeneity between the studies in the ablation technique and the mapping systems used. With the small patient numbers, there is no way to delineate which mapping system will be optimal for this patient group.

\section{Conclusion}

The studies outlined above suggest catheter ablation is an effective and durable long-term therapeutic strategy for postmyocarditis patients with VT, with limited relapse rates and very low complication rates. This early data suggests that ablation may be a useful adjunct to medical therapy and ICD implantation, to reduce the burden of polypharmacy or ICD shocks. However, the data so far is limited by small patient numbers and heterogeneity between studies. Larger randomised-controlled trials with standardised treatment and long follow-up are required to compare CA versus conventional treatment in the post-acute myocardial phase.

Supplementary Information The online version contains supplementary material available at https://doi.org/10.1007/s42399-022-01137-w.

Author Contribution EA: design of the manuscript, literature search, data analysis and interpretation, preparation and writing of the manuscript; DF: literature search and writing the manuscript; AB: data analysis; CS: writing and editing the manuscript; WYL: editing the manuscript; GS: editing the manuscript; NA: editing the manuscript; AG: editing the manuscript; NP: conception and design of the manuscript, critical revision of the article. All authors have read and approved the manuscript.

Data Availability Not applicable.

Code Availability Not applicable.

\section{Declarations}

Ethics Approval Not applicable.

Conflict of Interest The authors declare no competing interests.

\section{References}

1. Caforio AL, Pankuweit S, Arbustini E, Basso C, Gimeno-Blanes J, Felix SB, et al. Current state of knowledge on aetiology, diagnosis, management, and therapy of myocarditis: a position statement of the European Society of Cardiology Working 
Group on Myocardial and Pericardial Diseases. Eur Heart J. 2013;34(2636-48):48a-48d.

2. Tschope C, Ammirati E, Bozkurt B, Caforio ALP, Cooper LT, Felix SB, et al. Myocarditis and inflammatory cardiomyopathy: current evidence and future directions. Nat Rev Cardiol. 2020.

3. Peretto G, Sala S, Rizzo S, De Luca G, Campochiaro C, Sartorelli S, et al. Arrhythmias in myocarditis: state of the art. Heart Rhythm. 2019;16:793-801.

4. European Heart Rhythm Association, Heart Rhythm Society, Zipes DP, Camm AJ, Borggrefe M, Buxton AE, et al. ACC/AHA/ ESC 2006 guidelines for management of patients with ventricular arrhythmias and the prevention of sudden cardiac death: a report of the American College of Cardiology/American Heart Association Task Force and the European Society of Cardiology Committee for Practice Guidelines (Writing Committee to Develop Guidelines for Management of Patients With Ventricular Arrhythmias and the Prevention of Sudden Cardiac Death). J Am Coll Cardiol. 2006;48:e247-346.

5. Muratore CA, Baranchuk A. Current and emerging therapeutic options for the treatment of chronic chagasic cardiomyopathy. Vasc Health Risk Manag. 2010;6:593-601.

6. Vaseghi M, Hu TY, Tung R, Vergara P, Frankel DS, Di Biase L, et al. Outcomes of catheter ablation of ventricular tachycardia based on etiology in nonischemic heart disease: an international ventricular tachycardia ablation center collaborative study. JACC Clin Electrophysiol. 2018;4:1141-50.
7. Dello Russo A, Casella M, Pieroni M, Pelargonio G, Bartoletti S, Santangeli P, et al. Drug-refractory ventricular tachycardias after myocarditis: endocardial and epicardial radiofrequency catheter ablation. Circ Arrhythm Electrophysiol. 2012;5:492-8.

8. Berte B, Sacher F, Cochet H, Mahida S, Yamashita S, Lim H, et al. Postmyocarditis ventricular tachycardia in patients with epicardial-only scar: a specific entity requiring a specific approach. J Cardiovasc Electrophysiol. 2015;26:42-50.

9. De Cobelli F, Pieroni M, Esposito A, Chimenti C, Belloni E, Mellone R, et al. Delayed gadolinium-enhanced cardiac magnetic resonance in patients with chronic myocarditis presenting with heart failure or recurrent arrhythmias. J Am Coll Cardiol. 2006;47:1649-54.

10. Peretto G, Sala S, Basso C, Rizzo S, Radinovic A, Frontera A, et al. Inflammation as a predictor of recurrent ventricular tachycardia after ablation in patients with myocarditis. J Am Coll Cardiol. 2020;76:1644-56.

11. Maccabelli G, Tsiachris D, Silberbauer J, Esposito A, Bisceglia C, Baratto F, et al. Imaging and epicardial substrate ablation of ventricular tachycardia in patients late after myocarditis. Europace. 2014;16:1363-72.

Publisher's Note Springer Nature remains neutral with regard to jurisdictional claims in published maps and institutional affiliations. 